\title{
Salvia divinorum and Salvinorin A: An Update on Pharmacology and Analytical Methodology
}

\begin{abstract}
Salvia divinorum (Lamiaceae) has been used for centuries by the Mazatecan culture and has gained popularity as a recreational drug in recent years. Its potent hallucinogenic effects seen in case reports has triggered research and led to the discovery of the first highly selective non-nitrogenous $\kappa$ opioid receptor agonist salvinorin A. This review critically evaluates the reported pharmacological and toxicological properties of S. divinorum and one of its major compounds salvinorin A, its pharmacokinetic profile, and the analytical methods developed so far for its detection and quantification. Recent research puts a strong emphasis on salvinorin A, which has been shown to be a selective opioid antagonist and is believed to have further beneficial properties, rather than the leaf extract of S. divinorum. Currently animal studies show a rapid onset of action and short distribution and elimination half-lives as well as a lack of evidence of short- or long-term toxicity. Salvinorin A seems to be the most promising approach to new treatment options for a variety of CNS illnesses. However, many further investigations are necessary to fully un-
\end{abstract}

derstand and elucidate the various medicinal properties of the plant itself and to provide the legislative authorities with enough information to cast judgement on S. divinorum.

\section{Key words}

Salvia divinorum - Lamiaceae - depression - opioid antagonist . pharmacokinetics · pharmacology · toxicology · salvinorin A

\section{Abbreviations}

CNS: central nervous system

FST: forced swimming test

i.t. $\quad$ intrathecal

KOR: $\quad$ kappa opioid receptor

LSD: lysergic acid diethylamide

norBNI: norbinaltorphimine

p.o.: per os

s.c.: subcutaneous

ssp.: $\quad$ subspecies

\section{Introduction}

Salvia divinorum L. (Lamiaceae), or its more common name Diviner's sage, was used for traditional and ritual purposes for centuries in the Mazatecan culture. The plant belongs to the Lamiaceae (mint) family and has been classified by Epling and Jativa in 1962 [1]. The Mazatec used the plant as a psychoactive ingredient and believe that it is the reincarnation of the virgin Mary (the plant is often referred to as ska Maria, ska Pastora, hojas de Maria, or hojas de Pastora) [2]. Three routes of administration are reported for the traditional use of the leaves: (i) mastication and swallowing of the leaves, (ii) crushing the leaves to extract a juice and drinking the beverage, (iii) smoking the leaves [3]. Inhalation as suggested by the third traditional route of administration and chewing on the leaves are the most common routes of administration for recreational use in the United States [4] and Europe [5], making it a very 
potent and legal substitute for marijuana (Cannabis sativa L., Cannabaceae). The psychoactive effect of the plant is much more potent when smoked and lasts for about 15 minutes, as the ingestion of a juice or concentrate can prolong the effect up to one hour with an overall lesser potency [6]. Due to its high psychoactive and hallucinogenic properties, the Drug Enforcement Administration is closely monitoring $S$. divinorum with a possibility to place it in schedule 1 [6]. However, currently S. divinorum can be legally bought in the form of leaves or concentrates from the leaves and is grown in California [2]. Growing concern of parents due to a potential abuse of the plant and, on the other side, the scientific potential for the use of S. divinorum in the treatment of Alzheimer's disease, schizophrenia, and stimulant dependence [7] make it an ambiguous and controversial subject for discussion. Researchers who see the advantages for medicinal applications fear that classifying S. divinorum as a schedule 1 drug would slow or even halt promising research and applications. The proposed psychoactive compound of S. divinorum is a non-nitrogenic diterpenoid, salvinorin A (Fig. $\mathbf{1}$ ), whose molecular target seems to be the $\kappa$ opioid receptor (KOR). KOR agonists have been linked with dose-dependent and reversible subject/interceptive effects such as sedation, dysphoria, and psychotomimetic effects [8]. Besides the endogenous ligand at the KOR, dynorphin, some synthetic agonists have been shown to produce hallucinogenic effects similar to that of salvinorin A. However, so far the literature is lacking a scientific explanation as to how salvinorin A reaches the CNS after smoking, ingestion, or buccal absorption.

This article gives a brief review on the pharmacology of the salvinorins and other natural or synthetic diterpenes derived from Salvia divinorum, methods of detection for salvinorin A, its proposed pharmacokinetics, and metabolic fate.

\section{Pharmacological Activities}

All plants found in the sage genus (Salvia ssp.) possess a wide range of pharmacological activities, including sedative, hypnotic, muscle relaxant, analgesic, anticonvulsant, and neuroprotective effects [9]. So far only Salvia divinorum has been shown to exert hallucinogenic activities - and to this point this is the most scientifically investigated pharmacological activity. Although salvinorin A is thought to be the main psychoactive compound in $S$. divinorum, there may be other compounds with certain pharmacological activities that are as yet unexplored. Therefore, this review focuses on the psychopharmacological activities of salvinorin A as well as some semi-synthetic derivatives that have been developed for structure-activity relationship studies.

\section{Salvinorin A}

Salvinorin A has been shown to be a strong and selective agonist on the KOR [10], [11] by screening 50 distinctive systems involving receptors, transporters, and ion channels. Its distinctive profile is, however, different from that of the classic hallucinogen lysergic acid diethylamide (LSD). So far, salvinorin A shows high affinity only for KOR's both in vitro and in vivo which seems to follow a novel method of binding and activation not involving ionic interactions but possibly involving hydrophobic interaction. Due to the lack of a strong salt bridge and the multiplicity of hydrogen bonding and lipophilic binding sites in the KOR, it is likely that binding of salvinorin A does not follow only one single model [12]. The aforementioned discriminative stimulus effect was blocked by the $\kappa$ selective antagonist quadazocine, but not by the $\kappa$ selective antagonist GNTI [13] in vivo following intraperitoneal injection of salvinorin $A$. This suggests that the receptor binding site might be distinguishable by these two antagonists but, as yet, no research has been done to elucidate this behavior. The binding efficacy results in a full agonism on the KOR with a similar potency to that of the endogenous peptide ligand dynorphin $A$. The same research group recently published an in vivo study following intravenous injection of salvinorin A to rhesus monkeys [14] showing a dose-dependent increase in prolactin levels. This supports the influence on neuroendocrine functions and demonstrates that salvinorin $\mathrm{A}$ is able to readily cross the blood-brain barrier. The effect was antagonized by nalmefene, a $\mu$ and $\kappa$ opioid receptor antagonist, but not by ketanserin, a selective 5- $\mathrm{HT}_{2}$ antagonist, supporting the KOR selectivity of salvinorin A in exhibiting the observed neuroendocrine effects.

In vivo tests with salvinorin A showed a variety of results, some of them are unconclusive or incompatible with the in vitro data. Systemic administration of salvinorin A to rats showed an elevation in intracranial self-stimulation levels, which is explained as a depressive-like effect [15]. In addition, KOR antagonists exerted an antidepressant-like activity in the forced swimming test (FST) with rats [16], which is a well-established rodent behavior model for the evaluation of antidepressant drug effects [17]. Recently, one research group observed a depressant-like effect in the forced swimming test after intraperitoneal (i.p.) administration of salvinorin A in rats [18]. Salvinorin A was evaluated over a dose range from 0.125 to $2.0 \mathrm{mg} / \mathrm{kg}$ against a vehicle control (75\% DMSO). In addition, locomotor activity and intracranial self-stimulation were tested, and levels of serotonin and dopamine were analyzed using microdialysis probes in vivo [18]. While salvinorin A caused an increase in immobility time in the FST consistent with a depressive-like behavior and the results of intracranial self-stimulation were in agreement with previous results, it did not influence locomotor activity. In a concentration of $1 \mathrm{mg} / \mathrm{kg}$, salvinorin A caused consistent and long-lasting decreases in dopamine levels in the nucleus accumbens. However, no water control was used in these experiments to evaluate possible effects of DMSO and salvinorin A was injected intraperitoneally. It has been shown by Lukas et al. [19] that compounds administered i.p. are absorbed primarily through the portal circulation and, therefore, must pass through the liver before reaching other organs. The authors also could show that drugs are metabolized very differently depending on whether they are administered i.p. or by another parental route. Thus, the total amount of salvinorin A that gets absorbed might be higher after intraperitoneal injection than after oral application and therefore effects might have been detected which would not occur after oral application. Besides pharmacokinetic reasons, the extremely high concentration of the vehicle DMSO (75\%) adds another adulteration to the observed effect of salvinorin A.

Contrary to these observations from animal studies, there is one case report of the antidepressant effects of S. divinorum in man [20], which showed significant improvement of a patient who was smoking S. divinorum on a regular basis using a clinical depression scale rating. This might either be indicative of a metab- 
olism of salvinorin A or of other compounds in the plant that exhibit an antidepressant activity. If salvinorin $A$ is metabolized to an either active or inactive metabolite, then the binding affinity and the pharmacological profile studied in vitro are at jeopardy. A further study evaluated the effect of products of the in vitro autoxidation of salvinorin A. The main product of the neutral fraction after basic cleavage with $1 \mathrm{M} \mathrm{KOH}$ in methanol was an enedione. The major product in the base-soluble fraction was the 1,2-secotriester. Both the enedione and 1,2-secotriester were inactive on the $\sigma$ and $\mu$ receptors. In contrast to the enedione, the 1,2-secotriester had a weak affinity for the KOR. The study suggested that the 2-alkoxy function in salvinorin A seems not to be essential for the binding of salvinorin A to the KOR but that modification of this functional group alters the affinity to the receptor [21].

Lee at al. [22] evaluated the binding affinities for several compounds isolated from S. divinorum leaves using a hexane, acetone, and methanol extraction procedure. The acetone fraction showed the highest affinity toward the human KOR receptor by competitively inhibiting [23] the diprenorphine binding to the human KOR in Chinese hamster ovary cells by more than $50 \%$. The compounds that showed the highest affinity ranging from highest to lowest were salvinorin $\mathrm{A}$, salvinorin $\mathrm{B}$, divinatorin $\mathrm{D}$, and salvinorin G (Fig. 1) [24].

Another study evaluated the effects of salvinorin A given intraperitoneally via microdialysis on dopamine levels in two different brain regions (namely the caudate putamen and the nucleus accumbens) in mice as well as the effects of salvinorin A on locomotor activity and the conditional place aversion test for hallucinogenic effects [25]. Salvinorin A decreased the dopamine levels by 30 to $70 \%$ in the caudate putamen at a dose of 1 and $3.2 \mathrm{mg} /$ $\mathrm{kg}$, respectively. Surprisingly and unlike other KOR agonists, it did not cause a significant decrease in dopamine levels in the nucleus accumbens, which is in disagreement with other results [18] mentioned before. The two additional behavioral experiments revealed a positive conditioned place aversion and a decrease in locomotor activity for salvinorin A in both concentrations used to evaluate brain dopamine levels, which were all blocked by a KOR antagonist, norBNI. This is in agreement with the results from the locomotor activity of salvinorin A on inverted screen behavior in mice [26]. Salvinorin A in a dose of $2 \mathrm{mg} / \mathrm{kg}$ given i.p. significantly prolonged the time interval to climb the inverted screen. This reduction in locomotor activity was antagonized by norBNI and the unselective opioid receptor antagonist naloxone. One limitation of this experiment was the poor solubility for salvinorin A in a mixture of ethanol, organic solubilizers, and water as stated by the authors. This finding links in vitro results with in vivo observations and may explain some effects of the binding of salvinorin A to the KOR.

KOR agonists are known for their antinociceptive properties [27], which have been shown across a variety of rodents and non-humane primates. As salvinorin A is a highly selective KOR agonist, several publications investigated the analgesic properties in vitro and in vivo in rodents [28], [29], [30]. Results from a variety of antinociceptive experiments (e.g., hot plate test, tail flick test, acetic acid abdominal constriction) are inconclusive as salvinorin A showed consistently analgesic effects in one publication, but

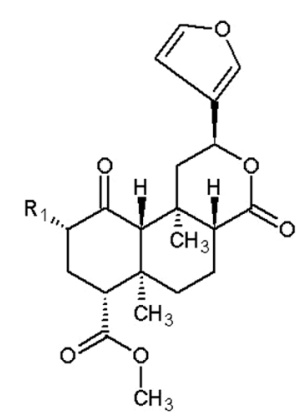

$\mathrm{R}_{1}: \mathrm{OCOCH}_{3}=$ Salvinorin $\mathrm{A}$ $\mathrm{R}_{1}: \mathrm{OH}=$ Salvinorin $\mathrm{B}$
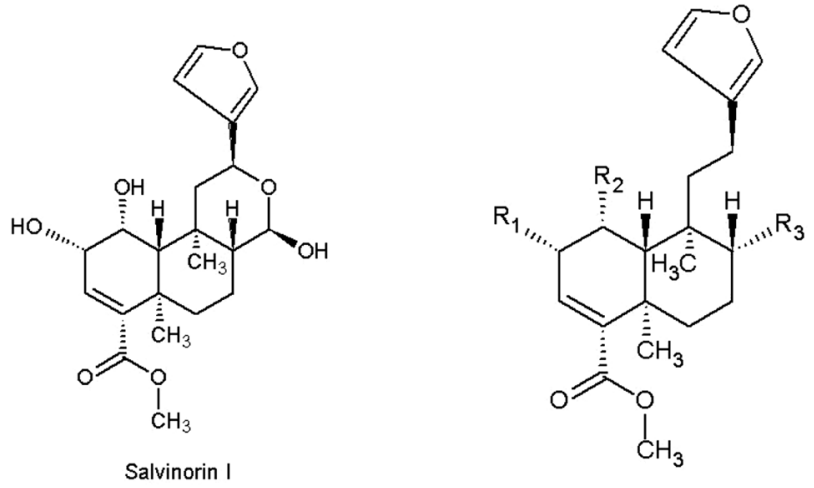

$\mathrm{R}_{1}: \mathrm{H} ; \mathrm{R}_{2}: \mathrm{OH} ; \mathrm{R}_{3}: \mathrm{CH}_{3}=$ Divinatorin $\mathrm{A}$ $\mathrm{R}_{1}: \mathrm{H} ; \mathrm{R}_{2}: \mathrm{OH} ; \mathrm{R}_{3}: \mathrm{CH}_{2} \mathrm{OH}=$ Divinatorin $\mathrm{B}$ $\mathbf{R}_{1}: \mathbf{H} ; \mathbf{R}_{2}: \mathbf{H} ; \mathbf{R}_{3}: \mathrm{CH}_{2} \mathrm{OCOCH}_{3}=$ Divinatorin $\mathrm{C}$ $\mathrm{R}_{1}: \mathrm{OH} ; \mathrm{R}_{2}: \mathrm{OH} ; \mathrm{R}_{3}: \mathrm{CH}_{2} \mathrm{OH}=$ Divinorin $\mathrm{F}$

Fig. 1 Structures of salvinorins $\mathrm{A}-\mathrm{H}$, and $\mathrm{I}$, divinorin $\mathrm{F}$, and divinatorins $A-C$.

failed to exert activity in the abdominal constriction test in the other. In contrast with two selective KOR agonists, salvinorin A did not effect antiscratching activity and antinociception in mice after subcutaneous administration [28], which the authors explain by a possible metabolism to inactive metabolites or concurrent actions at other receptors or ion channels. However, if salvinorin A would be metabolized to inactive metabolites then there must be other compounds in the plant material with hallucinogenic properties that do not interfere with the KOR. Contrary to these observations, another group was able to show a profound antinociceptive effect of salvinorin A following intrathecal injection [30]. This pain-reducing effect was antagonized with nor-binaltorphimine, a selective KOR antagonist, but not by beta-funaltrexamine (selective $\mu$ opioid receptor antagonist) or naltrindole (selective $\delta$ opioid receptor antagonist), supporting the high selectivity and specificity of salvinorin A on the KOR. One possible difference between the experiments could be associated to route of administration (s.c., p.o., and i.t.), delay until acetic acid exposure, and measurement interval. One indicator for a positive antinociception is that one group used three different experiments to evaluate the analgesic properties of salvinorin A and consistently got positive results [29]. 
One research group investigated the traditional use of $S$. divinorum as a treatment for abdominal swelling and diarrhea [31]. In this study, for the first time the whole leaf extract was evaluated in its ability to inhibit evoked contractions in an isolated guinea-pig ileum. The plant extract was prepared containing no salvinorin $\mathrm{A}$ to a concentration of $1.6 \%$ salvinorin $\mathrm{A}$ by addition of the pure compound. The extract without salvinorin A was not able to abolish or reduce evoked contractions while the salvinorin A-containing extract inhibited the contractions in a dose-dependent fashion ( $\mathrm{IC}_{50}$ values were $7504 \mathrm{ng} / \mathrm{mL}$ and 1.72 $\mathrm{ng} / \mathrm{mL}$, respectively). Additional experiments to evaluate the inhibitory effect of the salvinorin A-containing extract revealed that only KOR antagonists (namely naloxone and nor-binaltorphimine) evoked contractions were inhibited, while other receptors were not effected. Both the salvinorin A-containing extract as well as salvinorin $A$ alone were not able to effect contractions evoked by exogenous acetylcholine stimulation. In addition, the same experiments were conducted using pure salvinorin A and resulted in the specific blocking of contractions evoked by naloxone and nor-binaltorphimine supporting the current KOR selectivity of salvinorin A.

\section{Other diterpenes from Salvia divinorum}

In recent years more diterpenes from Salvia divinorum have been described. Salvinorins C - I [32], [33], [34], divitanorins A-F [35], [34], salvinicins A and B [36], and salvidivins A-D (Fig. 2) [34]. One group also reported on the biological activity of the two new neoclerodane diterpenes salvinicin A and B. Both compounds were screened using the $\left.{ }^{35} \mathrm{~S}\right] \mathrm{GTP} \gamma \mathrm{S}$ assay and exhibited partial $\kappa$ agonist and $\mu$ agonist activity, respectively. It is still unclear if any of these new diterpenes play a significant role in the hallucinogenic properties of S. divinorum.
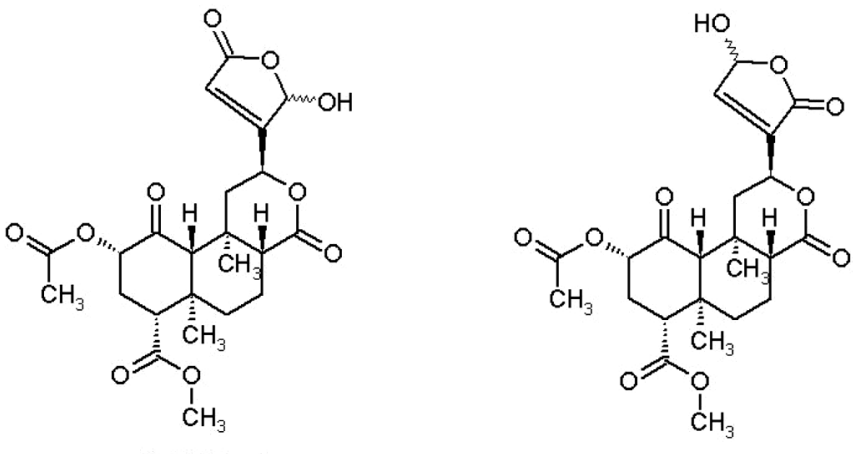

Salvidivin A
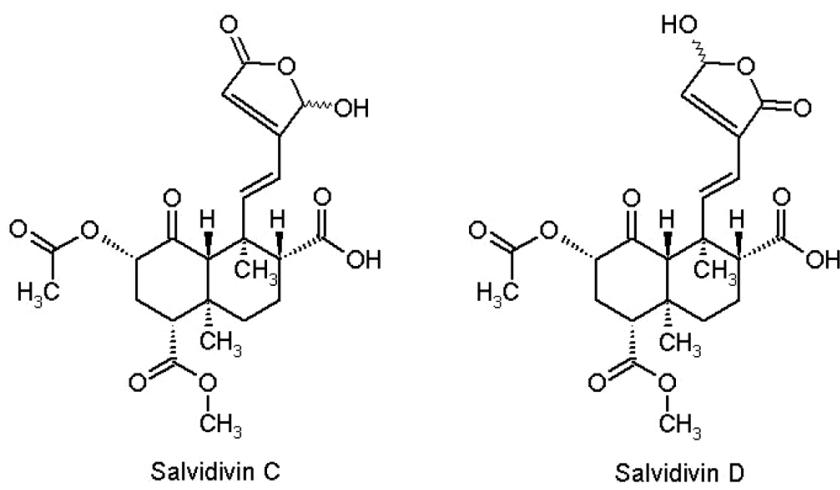

Fig. 2 Structures of salvidivins A-D.

\section{Semi-synthetic derivatives}

In a binding study with several synthesized analogues of salvinorin $A$ it was shown that alterations in the furan ring and substitution of the side chains on the $\alpha$-acetylated alcohol and the neighboring ketone have a strong effect on the binding efficacy and potency. While longer carbohydrate chains lower the binding affinity only marginally, a substitution at the ketone side results in a loss of affinity to the KOR as does a hydrogenation of the furan ring [37]. Harding et al. [38] found that, generally, replacement of the furan ring decreased KOR affinity. Of the functional groups used $\mathrm{N}$-sulfonylpyrrole was more tolerable than 4carbomethoxyoxazole. Interestingly, the mesylate at the $\alpha$ acetylated alcohol caused an increase in binding affinity. Further screening of nine different ester derivatives in the 2 position of salvinorin A suggested that only the 2-proprionate has affinity for the human KOR in a cell-based model in submicromolar concentrations. Furthermore, no significant affinities for any of the other receptor types tested including serotonergic, dopaminergic, muscarinic, adrenergic, cannabinoid, or $\sigma$ receptors were found. Interestingly, another compound believed to be important in mediating the effects of S. divinorum (salvinorin B) was inactive at human KOR's [39].

These findings are confirmed by Harding et al. [38] who also showed the proprionate and mesylate to be selective KOR agonists. Furthermore, the benzoate was identified as the first nonnitrogeneous $\mu$ receptor agonist, suggesting that the introduction of an aromatic functional group at the 2 position of salvinorin $\mathrm{A}$ increases the $\mu$ receptor affinity [38]. In a related study by Tidgewell et al. [40] several analogues of salvinorin A and a similar structure, herkinorin, were created and used as probes to further understand neoclerodane binding preferences in opioid systems. The authors looked at four main trends; elongation of the carbon chain, substitution using aromatic sulfonyl groups, halogenated aromatics, and different carbon spacers between the carbonyl and a phenyl ring. Increasing the carbon chain by two or more carbon atoms generally decreased the affinity at the KOR. The single carbon spaced phenyl analogue decreased the affinity at the KOR compared to herkinorin as well as a decrease for $\mu$ receptors. The additional methylene spacer increased affinity for both KOR and $\mu$ receptors. The analogues with halogenated aromatic groups exhibited a decrease in $\delta$ receptor affinity and subsequently increased selectivity at $\kappa$ and $\mu$. Lastly, the addition of the aromatic sulfonyl groups had no effect of KOR affinity.

\section{Pharmacokinetics of Salvinorin A}

Siebert et al. [3] tested various methods of leaf and salvinorin intake by volunteers to determine the site of absorption, effects, and dosage. It was found that extended exposure (10 $\mathrm{min})$ to the oral mucosa produced psychoactive effects in all volunteers, while quick swallowing and rinsing of leaves produced no effects at all. The authors concluded that the gastrointestinal system breaks down the psychoactive compound and that the leaves must be chewed or held in the mouth to produce hallucinations. Interestingly, 20 volunteers were given capsules of salvinorin A, which produced no effects, reinforcing the hypothesis that salvinorin is inactivated by gastrointestinal absorption. Because salvinorin $\mathrm{A}$ is not water soluble, injection was not tested by the au- 
thors [3]. It is assumed that traditionally the herb is consumed either by chewing the fresh leaves or by drinking the juices or teas of the leaves [41]. The effects of the herb when consumed in this way depend on the absorption of salvinorin A through the oral mucosa before the herb is swallowed. In this context it also has been discussed that chewing the leaves might not be the traditional method of ingesting Salvia divinorum because of the extremely bitter flavor of the leaves [42]. Inhalation - though not used traditionally - of the vaporized salvinorin A proved to be the most efficient method to produce hallucinations which occurred within 30 seconds, rather than the $10-15$ minutes observed after oral ingestion [3]. However, systematic pharmacokinetic studies with the whole herb, especially with its traditional dosage forms such as juices or teas have not been performed so far.

To date, only one pharmacokinetic study has been performed in non-human primates administered salvinorin A intravenously [43]. The pharmacokinetic properties of salvinorin A were studied using four rhesus monkeys, which were administered $0.032 \mathrm{mg} / \mathrm{kg}$ salvinorin A, the dose that was previously found to exert behavioral responses after subcutaneous injection. Samples were drawn before (baseline) and at $0,1,5,15,30$, and 60 minutes following i. $v$. injection of salvinorin $\mathrm{A}$. The areas under the concentration-time curve (AUC) as well as the half-lives for distribution and elimination $\left(\mathrm{t}_{1 / 2}\right)$ have been calculated according to standard methods (although one limitation was to use only two time points for the calculation of the half-lives). Sedationlike effects were seen in all animals immediately after intravenous administration of $0.032 \mathrm{mg} / \mathrm{kg}$ salvinorin A which lasted for about 15 minutes. Although this study was only performed in two male and two female subjects, there seems to be a gender-related difference in the distribution and elimination halflives, where males have a faster distribution half-life compared to females and both a shorter elimination half-life and AUC - in both cases almost only half of that of the females (Table $\mathbf{1}$ ). This gender difference has been seen in other drugs acting on the opioid or nociceptive receptor system [44].

This study has only limited explanations for the full pharmacokinetic spectrum of salvinorin A as excretion via the bile and metabolism to active or inactive metabolites has not been discussed. In addition, the pharmacokinetics of salvinorin A may be influenced if taken with the whole leaf extract of Salvia divinorum as has been shown with many other plant derived extracts before [45]. Currently, the literature lacks detailed insight on the metabolism of salvinorin A and how it reaches its binding site in the brain. Further investigations of the complete pharmacokinetic profile of salvinorin A and the leaf extract of Salvia divinorum are therefore necessary.

Table 1 Pharmacokinetic parameters for salvinorin A in relation to gender. Data adapted from [43]

\begin{tabular}{lllc}
\hline & $\begin{array}{l}\text { Distribution half-life } \\
\text { [min] }\end{array}$ & $\begin{array}{l}\text { Elimination half-life } \\
\text { [min] }\end{array}$ & $\begin{array}{l}\text { AUC } \\
{[\mathbf{n g} \times \mathbf{m i n} / \mathbf{m L}]}\end{array}$ \\
\hline Male & rapid & $37.9 \pm 5.6$ & $572 \pm 133$ \\
\hline Female & $0.95 \pm 0.2$ & $80.0 \pm 13.1$ & $1087 \pm 46$ \\
\hline
\end{tabular}

\section{Toxicology}

A potential toxicity is usually linked to the use of potent psychoactive drugs [46], [47], [48] which affects different brain regions resulting in a degradation of brain tissue. So far only one study has examined possible toxic side effects of salvinorin A in rodents [49]. In a two-week chronic treatment study, no or little histological differences were observed after high doses of salvinorin A when given intraperitoneally. As the study was only conducted for two weeks and no biochemical markers were used to investigate toxic effects, potential chronic toxicity linked to salvinorin A cannot be excluded. To the present, no study has investigated a chronic or acute toxicity of the leaf extract of Salvia divinorum. Another factor might be a possible biotransformation that leads to the detoxification of potential toxins. Reports from human case studies have not mentioned any toxic side effects linked to the use of S. divinorum as a recreational drug [50]. However, these case reports are limited in their ability to provide sufficient information.

\section{Analytical Procedures}

The detection of drugs with an abusive potential plays an important role in legal investigations. The implication of the use of Salvia divinorum is a special case in the United States and many other countries as the recreational use of the plant and any extracts or derived products are not classified as illegal or illicit. In the United States, S. divinorum was placed on a list of drugs labeled "with concern" by the Drug Enforcement Agency (DEA) resulting in no legal implications. Recently, the DEA has placed this plant on its watch list for possible scheduling. This substance has been banned in 5 states (Delaware, Louisiana, Missouri, Oklahoma, and Tennessee) and two states (New Jersey and New York) are currently formulating legislature on Salvia divinorum. Some attempts have been made to prohibit the legal sale at the national level, but this has been met with great resistance. Other countries like Australia, Denmark, and Finland, however, have placed S. divinorum on the list of illicit drugs prohibiting any use due to unknown health risks and an unknown addictive potential [51]. Therefore, the establishment of analytical methods for the detection of salvinorin $\mathrm{A}$ as the proposed hallucinogenic compound and its metabolites in body fluids is a useful approach to aid the medico-legal investigator.

To date, there are two analytical methods for the detection in body fluids [52], [53] and four methods for the detection and localization of salvinorins in plant material [54], [55], [56], [57]. One of the methods for detection in body fluids had the serious drawback that blood samples were ex vivo spiked with salvinorin A rather than drawing blood after the systemic administration of the substance. The other method had a sample size of two volunteers smoking a fixed amount of plant material. Unfortunately, only urine and saliva samples could be collected as the subjects refused blood collection after the administration.

The first method [54] for the identification of the plant $S$. divinorum uses a liquid chromatography method coupled with an electrospray ionization multistage ion trap mass spectrometry $\left(\mathrm{LC} / \mathrm{ESI}-\mathrm{IT}_{\mathrm{T}} \mathrm{MS} \mathrm{N}^{\mathrm{n}}\right)$. The structure of salvinorin A does not allow 
the usual extraction method with a high $\mathrm{pH}$ value for gas chromatography/mass spectrometry (GC/MS) or liquid chromatography/ultraviolet (LC/UV) detection as the ester group is prone to hydrolysis. The plant leaves were extracted with acetonitrile/water (50:50), filtered, and injected in the LC/MS. Standards of a variety of salvinorins and divinatorins were dissolved in methanol/water (50:50) and injected. The following limits of detection (LOD) were obtained for salvinorins A (LOD range: 3-367 ng/ $\mathrm{mL}$ ), B (LOD range: $7-1144 \mathrm{ng} / \mathrm{mL}$ ), C (LOD range: $2-221 \mathrm{ng} /$ $\mathrm{mL}$ ), and $\mathrm{D}$ (LOD range: $9-128 \mathrm{ng} / \mathrm{mL}$ ) using $\mathrm{LC} / \mathrm{MS}^{2}$, LC/MS ${ }^{3}$, GC/MS, and LC/UV methods for comparison.

This method can prove useful in identifying the plant, possible dilutions with other plants, and the origin of the variety of $S$. divinorum as the Mazatecan sample is higher in salvinorin A while a Hawaiian sample contained more salvinorin B. In addition, the multistage mass spectrometry elucidated a very detailed fragmentation pattern for salvinorins and divinatorins, which may serve as a reference for future analytical methods.

Recently, another method for the detection and quantification of salvinorin $\mathrm{A}$ has been established and used to screen commercially available leaves and a leaf extract from $S$. divinorum for their salvinorin A contents [55]. Interestingly, the claimed potencies stated on the products could not be recovered quantitatively from the analysis. Instead, three out of five samples contained adulterations of caffeine or vitamin $\mathrm{E}$ and all five samples had significantly lower salvinorin A amounts than stated (ranging from $1-16 \%$ of the claimed amount). The analytical method used for the quantification of salvinorin A was an HPLC-UV/Vis system using an isocratic elution with acetonitrile: water (45:55) and detection at $208 \mathrm{~nm}$ [56]. Adulterations were identified by thin layer chromatography-GC/MS. These commercially available samples of $S$. divinorum still seem to show the desired hallucinogenic effect even though the salvinorin A concentration is regarded to be subpotent, as stated by the authors.

A simple method for the localization of salvinorins in the plant has been established using thin layer chromatography [57]. This method used chloroform swabs to extract salvinorins and evaluated the distribution pattern of the terpenoids in leaf, stem, rachis, bract, calyx, and corolla of mature plants. In general, the glandular (secretory) trichomes of the abaxial, young leaves show the highest salvinorin content while the roots, internal stem tissue, cotyledons, and corolla are absent of salvinorins. The author concluded that salvinorins are secreted through the peltate glandular trichomes and that especially young leaves are rich in salvinorins.

The ex vivo analytical method used a high performance liquid chromatography method coupled with a mass spectrometry instrument (HPLC/MS). Blood and cerebrospinal fluid samples were taken from a male rhesus monkey never exposed to $S$. divinorum or salvinorins. Blood samples were kept at $37^{\circ} \mathrm{C}$ and spiked with $1900 \mathrm{ng} / \mathrm{mL}$ salvinorin A. Over one hour six samples were drawn, cooled, and the plasma separated for analysis. Human urine was spiked with salvinorin A over a range of concentrations between 1 and $500 \mathrm{ng} / \mathrm{mL}$. An internal standard was used and a standard curve between 2 and $1000 \mathrm{ng} / \mathrm{mL}$ salvinorin A analyzed. All samples were subjected to solid phase extraction before injection. The limit of quantification was found to be $2 \mathrm{ng} /$ $\mathrm{mL}$ according to FDA guidelines. However, the paper did not present any results on the cerebrospinal fluid analysis other than the recovery rate nor is the urine used for quantification purposes, but rather for intra- and interday precision and accuracy. The main purpose mentioned in the discussion was to determine the degree of metabolism in the formation of salvinorin $B$ as the main metabolite of salvinorin A. Within 30 minutes following incubation of blood samples, the concentration of salvinorin $B$ exceeds that of salvinorin $A$. This suggests that salvinorin A is metabolized via blood esterases to form salvinorin B. This might give important hints to the clinical and forensic application and detection pattern for salvinorin A. If the main metabolite in vivo is indeed salvinorin $\mathrm{B}$, a method should be developed for quantification of salvinorin B in different body fluids like plasma, urine, saliva, and sweat after administration to humans.

The main route of administration for recreational use of $S$. divinorum is smoking the dried leaves. One analytical method established the detection of salvinorin A in plasma, urine, saliva, and sweat using a GC/MS method following extraction from biological matrices [53]. Method validation was accomplished over a range from 0.015 to $5 \mu \mathrm{g} / \mathrm{mL}$ for salvinorin A in different matrices with a precision and accuracy of at least $15 \%$. The limit of detection was $5 \mathrm{ng} / \mathrm{mL}$ and the limit of quantification was $15 \mathrm{ng} /$ $\mathrm{mL}$. Samples were analyzed from two volunteers after smoking $75 \mathrm{mg}$ of dried leaf material containing $0.58 \mathrm{mg}$ salvinorin A. Unfortunately, blood samples could not be collected due to a vivid hallucination stage starting 30 seconds after smoking and lasting for 15-20 minutes. After this period, both individuals refused to have blood samples drawn.

Even though this study was fairly small with only two subjects and blood samples could not be obtained, some important conclusions about the metabolism and excretion of salvinorin A can be drawn. For legal investigations, saliva seems to be a valid matrix for the analysis of salvinorin $A$ with the limitation that as yet no pharmacokinetic data are available and the possibility that residues from the plant material in the mouth cavern might be responsible for an overestimation of salvinorin A concentrations. The route of excretion is either via metabolism to salvinorin $B$ or other metabolites and via direct elimination into saliva (with the probability of reabsorption) and urine. As the limit of detection was set fairly low (5 ng), sweat does not seem to be the preferred route of elimination. In conclusion, the method developed in this publication is as yet the only in vivo assay for the detection and quantification of salvinorin A. Further improvement including the concomitant detection of the proposed metabolite salvinorin $\mathrm{B}$ is necessary to optimize and apply this method for forensic and legal applications.

\section{Conclusion}

The increasing recreational use of Salvia divinorum in various countries led both to a public concern and an interest in the plant and its activities. The epidemiology and use pattern of $S$. divinorum seems to be linked both to a recent popularity of the drug and its legal availability through various internet sources. However, there is as yet no detailed insight into the specific pop- 
ulation of recreational Salvia users. Research in the last ten years mainly focused on the hallucinogenic properties of the dried leaves leading to the identification of the specific KOR agonist salvinorin A. Structure-binding studies have shown that derivatives, semi-synthetic compounds, and other similar substances present in S. divinorum possess only little KOR activity and the structure is therefore highly specific with only small modifications allowing for similar or increased activity. Besides its hallucinogenic activity, salvinorin A has been shown to possess antinociceptive properties as well as depressant-like effects as seen with other KOR agonists. A microdialysis study looked at the influence of salvinorin A administration on dopamine levels in certain brain regions and the results suggest an influence on dopamine levels either in the caudate putamen or nucleus accumbens. This specificity is a deviation from the regular action of KOR's and further investigation is necessary to distinguish and explain this deviation. The whole plant extract showed a variety of pharmacological activities which have as yet not been further explored. The toxicological profile of salvinorin A and Salvia divinorum is sparse and further research needs to be conducted in order to reach conclusive results. The recreational use of $S$. divinorum has been reported to exert only small if any side effects, although the dose needs to be increased over time to get the same effect, which might be indicative of a potential addiction problem [51]. Two studies investigated the pharmacokinetics of salvinorin A in non-human primates after i. $v$. administration. Further investigations with a higher sample size are necessary to elucidate in-depth time-concentration profiles of salvinorin $\mathrm{A}$ and in addition more studies are needed with the whole plant extract. The results obtained should be regarded with caution as the route of administration differs from the preferred route by inhalation. An important issue regarding legal investigations that might arise within the next years in several countries is the establishment of a sensitive and practical applicable analytical method for the detection of salvinorin A or its metabolites in commonly analyzed body fluids. So far, only one GC/MS method has been established with the limitation of no obtained samples for blood analysis, a small sample size and period [53]. However, this method has been proven to be useful in evaluating the routes of elimination and the range of amounts to expect from different matrices. Further investigations into routes of elimination, metabolism to inactive or active metabolites and their pharmacokinetic properties, and longer post-exposure analysis of samples are necessary to institute an analytical method.

In conclusion, in order to make an educated and reasonable decision on whether Salvia divinorum and salvinorin A should be classified as illegal drugs, further research and case reports are necessary. A clinical application of S. divinorum should be considered if further investigations show conclusively a beneficial effect of the leaf extract in the treatment of a variety of mental disorders like depression, anxiety, Alzheimer's disease, or schizophrenia.

\section{References}

${ }^{1}$ Epling C, Jativa MC. A new species of Salvia from Mexico. Bot Mus Leafl Harv Univ 1962; 20: 75-6.
2 Sheffler DJ, Roth BL. Salvinorin A: the "magic mint" hallucinogen finds a molecular target in the kappa opioid receptor. Trends Pharmacol Sci 2003; 24: 107-9.

${ }^{3}$ Siebert DJ. Salvia divinorum and salvinorin A: new pharmacologic findings. J Ethnopharmacol 1994; 43: $53-6$.

${ }^{4}$ Valdes LJ 3rd. Salvia divinorum and the unique diterpene hallucinogen, Salvinorin (divinorin) A. J Psychoactive Drugs 1994; 26: 277-83.

${ }^{5}$ Giroud C, Felber F, Augsburger M, Horisberger B, Rivier L, Mangin P. Salvia divinorum: an hallucinogenic mint which might become a new recreational drug in Switzerland. Forensic Sci Int 2000; 112: 143 - 50.

${ }^{6}$ Halpern $\mathrm{JH}$. Hallucinogens and dissociative agents naturally growing in the United States. Pharmacol Ther 2004; 102: 131 -8.

${ }^{7}$ Prisinzano TE. Psychopharmacology of the hallucinogenic sage Salvia divinorum. Life Sci 2005; 78: 527 - 31 .

8 Bertea CM, Luciano P, Bossi S, Leoni F, Baiocchi C, Medana C et al. PCR and PCR-RFLP of the 5S-rRNA-NTS region and salvinorin A analyses for the rapid and unequivocal determination of Salvia divinorum. Phytochemistry 2006; 67: $371-8$.

${ }^{9}$ Imanshahidi M, Hosseinzadeh H. The pharmacological effects of Salvia species on the central nervous system. Phytother Res 2006; 20: 427 37.

${ }^{10}$ Roth BL, Lopez E, Beischel S, Westkaemper RB, Evans JM. Screening the receptorome to discover the molecular targets for plant-derived psychoactive compounds: a novel approach for CNS drug discovery. Pharmacol Ther 2004; 102: 99-110.

11 O'Connor KA, Roth BL. Screening the receptorome for plant-based psychoactive compounds. Life Sci 2005; 78: 506-11.

12 Yan F, Mosier PD, Westkaemper RB, Stewart J, Zjawiony JK, Vortherms TA et al. Identification of the molecular mechanisms by which the diterpenoid salvinorin A binds to kappa-opioid receptors. Biochemistry 2005; 44: 8643-51.

${ }^{13}$ Butelman ER, Harris TJ, Kreek MJ. The plant-derived hallucinogen, salvinorin $A$, produces kappa-opioid agonist-like discriminative effects in rhesus monkeys. Psychopharmacology (Berl) 2004; 172: 220-4.

${ }^{14}$ Butelman ER, Mandau M, Tidgewell K, Prisinzano TE, Yuferov V, Kreek MJ. Effects of salvinorin A, a \{kappa\}-opioid hallucinogen, on a neuroendocrine biomarker assay in non-human primates with high kappa\}-receptor homology to humans. J Pharmacol Exp Ther 2007; 320: 300-6.

15 Todtenkopf MS, Marcus JF, Portoghese PS, Carlezon WA Jr. Effects of kappa-opioid receptor ligands on intracranial self-stimulation in rats. Psychopharmacology (Berl) 2004; 172: 463-70.

${ }^{16}$ Mague SD, Pliakas AM, Todtenkopf MS, Tomasiewicz HC, Zhang Y, Stevens WCJr. et al. Antidepressant-like effects of kappa-opioid receptor antagonists in the forced swim test in rats. J Pharmacol Exp Ther 2003; 305: $323-30$.

${ }^{17}$ Thiebot MH, Martin P, Puech AJ. Animal behavioural studies in the evaluation of antidepressant drugs. Br J Psychiatry Suppl; 1992: 4450.

${ }^{18}$ Carlezon WA Jr, Beguin C, DiNieri JA, Baumann MH, Richards MR, Todtenkopf MS et al. Depressive-like effects of the kappa-opioid receptor agonist salvinorin A on behavior and neurochemistry in rats. J Pharmacol Exp Ther 2006; 316: 440-7.

${ }^{19}$ Lukas G, Brindle SD, Greengard P. The route of absorption of intraperitoneally administered compounds. J Pharmacol Exp Ther 1971; 178: $562-4$

${ }^{20}$ Hanes KR. Antidepressant effects of the herb Salvia divinorum: a case report. J Clin Psychopharmacol 2001; 21: 634-5.

${ }^{21}$ Munro TA, Goetchius GW, Roth BL, Vortherms TA, Rizzacasa MA. Autoxidation of salvinorin A under basic conditions. J Org Chem 2005; 70 : $10057-61$.

${ }^{22}$ Lee DY, Ma Z, Liu-Chen LY, Wang Y, Chen Y, Carlezon WA Jr et al. New neoclerodane diterpenoids isolated from the leaves of Salvia divinorum and their binding affinities for human kappa opioid receptors. Bioorg Med Chem 2005; 13: 5635-9.

${ }^{23}$ Kavvadias D, Monschein V, Sand P, Riederer P, Schreier P. Constituents of sage (Salvia officinalis) with in vitro affinity to human brain benzodiazepine receptor. Planta Med 2003; 69: 113-7.

24 Beguin C, Richards MR, Wang Y, Chen Y, Liu-Chen LY, Ma Z et al. Synthesis and in vitro pharmacological evaluation of salvinorin A analogues modified at $C(2)$. Bioorg Med Chem Lett 2005; 15: $2761-5$.

${ }^{25}$ Zhang Y, Butelman ER, Schlussman SD, Ho A, Kreek MJ. Effects of the plant-derived hallucinogen salvinorin A on basal dopamine levels in the caudate putamen and in a conditioned place aversion assay in 
mice: agonist actions at kappa opioid receptors. Psychopharmacology (Berl) 2005; 179: 551 -8.

${ }^{26}$ Fantegrossi WE, Kugle KM, Valdes LJ 3rd, Koreeda M, Woods JH. Kappa-opioid receptor-mediated effects of the plant-derived hallucinogen, salvinorin $A$, on inverted screen performance in the mouse. Behav Pharmacol 2005; 16: 627-33.

${ }^{27}$ Vonvoigtlander PF, Lahti RA, Ludens JH. U-50,488: a selective and structurally novel non-Mu (kappa) opioid agonist. J Pharmacol Exp Ther 1983; 224: 7-12.

${ }^{28}$ Wang Y, Tang K, Inan S, Siebert D, Holzgrabe U, Lee DY et al. Comparison of pharmacological activities of three distinct kappa ligands (Salvinorin A, TRK-820 and 3FLB) on kappa opioid receptors in vitro and their antipruritic and antinociceptive activities in vivo. J Pharmacol Exp Ther 2005; 312: 220 - 30.

${ }^{29}$ McCurdy CR, Sufka KJ, Smith GH, Warnick JE, Nieto MJ. Antinociceptive profile of salvinorin $A$, a structurally unique kappa opioid receptor agonist. Pharmacol Biochem Behav 2006; 83: 109-13.

${ }^{30}$ John TF, French LG, Erlichman JS. The antinociceptive effect of salvinorin A in mice. Eur J Pharmacol 2006; 545: 129-33.

${ }^{31}$ Capasso R, Borrelli F, Capasso F, Siebert DJ, Stewart DJ, Zjawiony JK et al. The hallucinogenic herb Salvia divinorum and its active ingredient salvinorin A inhibit enteric cholinergic transmission in the guinea-pig ileum. Neurogastroenterol Motil 2006; 18: 69-75.

32 Valdes LJ 3rd, Chang HM, Visger DC, Koreeda M. Salvinorin C, a new neoclerodane diterpene from a bioactive fraction of the hallucinogenic Mexican mint Salvia divinorum. Org Lett 2001; 3: 3935-7.

${ }^{33}$ Munro TA, Rizzacasa MA. Salvinorins D-F, new neoclerodane diterpenoids from Salvia divinorum, and an improved method for the isolation of salvinorin A. J Nat Prod 2003; 66: $703-5$.

${ }^{34}$ Shirota O, Nagamatsu K, Sekita S. Neo-clerodane diterpenes from the hallucinogenic sage Salvia divinorum. J Nat Prod 2006; 69: 1782-6.

35 Bigham AK, Munro TA, Rizzacasa MA, Robins-Browne RM. Divinatorins $\mathrm{A}-\mathrm{C}$, new neoclerodane diterpenoids from the controlled sage Salvia divinorum. J Nat Prod 2003; 66: 1242 -4.

${ }^{36}$ Harding WW, Tidgewell K, Schmidt M, Shah K, Dersch CM, Snyder J et al. Salvinicins A and B, new neoclerodane diterpenes from Salvia divinorum. Org Lett 2005; 7: 3017-20.

${ }^{37}$ Munro TA, Rizzacasa MA, Roth BL, Toth BA, Yan F. Studies toward the pharmacophore of salvinorin A, a potent kappa opioid receptor agonist. J Med Chem 2005; 48: 345-8.

${ }^{38}$ Harding WW, Schmidt M, Tidgewell K, Kannan P, Holden KG, Dersch $\mathrm{CM}$ et al. Synthetic studies of neoclerodane diterpenes from Salvia divinorum: selective modification of the furan ring. Bioorg Med Chem Lett 2006; 16: 3170-4.

${ }^{39}$ Chavkin C, Sud S, Jin W, Stewart J, Zjawiony JK, Siebert DJ et al. Salvinorin $\mathrm{A}$, an active component of the hallucinogenic sage Salvia divinorum is a highly efficacious kappa-opioid receptor agonist: structural and functional considerations. J Pharmacol Exp Ther 2004; 308: $1197-203$.

${ }^{40}$ Tidgewell K, Harding WW, Lozama A, Cobb H, Shah K, Kannan P et al. Synthesis of salvinorin A analogues as opioid receptor probes. J Nat Prod 2006; 69: 914-8.

${ }^{41}$ Wasson RG. A new Mexican psychotropic drug from the mint family. Bot Mus Leafl Harv Univ 1962; 20: 163-93.
42 Valdes LJ 3rd, Diaz JL, Paul AG. Ethnopharmacology of ska Maria Pastora (Salvia divinorum, Epling and Jativa-M.). J Ethnopharmacol 1983; 7: $287-312$

${ }^{43}$ Schmidt MD, Schmidt MS, Butelman ER, Harding WW, Tidgewell K, Murry DJ et al. Pharmacokinetics of the plant-derived kappa-opioid hallucinogen salvinorin A in nonhuman primates. Synapse 2005; 58: $208-10$.

${ }^{44}$ Negus SS, Wurrey BA, Mello NK. Sex differences in thermal nociception and prostaglandin-induced thermal hypersensitivity in rhesus monkeys. J Pain 2004; 5: $92-103$.

45 Butterweck V, Lieflander-Wulf U, Winterhoff H, Nahrstedt A. Plasma levels of hypericin in presence of procyanidin B2 and hyperoside: a pharmacokinetic study in rats. Planta Med 2003; 69: 189-92.

46 Rold JF. Mushroom madness. Psychoactive fungi and the risk of fatal poisoning. Postgrad Med 1986; 79: $217-8$.

47 Scallet AC, Lipe GW, Ali SF, Holson RR, Frith CH, Slikker W Jr. Neuropathological evaluation by combined immunohistochemistry and degeneration-specific methods: application to methylenedioxymethamphetamine. Neurotoxicology 1988; 9: 529-37.

48 Tiongson J, Salen P. Mass ingestion of Jimson Weed by eleven teenagers. Del Med J 1998; 70: 471 -6.

${ }^{49}$ Mowry M, Mosher M, Briner W. Acute physiologic and chronic histologic changes in rats and mice exposed to the unique hallucinogen salvinorin A. J Psychoactive Drugs 2003; 35: 379-82.

${ }^{50}$ Schaper D. Legal, herbal hallucinogenic draws teens, critics. In: All things considered. United States of America: National Public Radio; 2006: 7 minutes 25 seconds..

51 Bucheler R, Gleiter CH, Schwoerer P, Gaertner I. Use of nonprohibited hallucinogenic plants: increasing relevance for public health. A case report and literature review on the consumption of Salvia divinorum (Diviner's Sage)? Pharmacopsychiatry 2005; 38: 1-5.

${ }^{52}$ Schmidt MS, Prisinzano TE, Tidgewell K, Harding W, Butelman ER, Kreek MJ et al. Determination of salvinorin A in body fluids by high performance liquid chromatography-atmospheric pressure chemical ionization. J Chromatogr B Analyt Technol Biomed Life Sci 2005; 818: $221-5$.

53 Pichini S, Abanades S, Farre M, Pellegrini M, Marchei E, Pacifici R et al. Quantification of the plant-derived hallucinogen Salvinorin A in conventional and non-conventional biological fluids by gas chromatography/mass spectrometry after Salvia divinorum smoking. Rapid Commun Mass Spectrom 2005; 19: 1649-56.

${ }^{54}$ Medana C, Massolino C, Pazzi M, Baiocchi C. Determination of salvinorins and divinatorins in Salvia divinorum leaves by liquid chromatography/multistage mass spectrometry. Rapid Commun Mass Spectrom 2006; 20: $131-6$.

55 Wolowich WR, Perkins AM, Cienki JJ. Analysis of the psychoactive terpenoid salvinorin A content in five Salvia divinorum herbal products. Pharmacotherapy 2006; 26: $1268-72$.

56 Gruber JW, Siebert DJ, Der Marderosian AH, Hock RS. High performance liquid chromatographic quantification of salvinorin A from tissues of Salvia divinorum Epling \& Ja'tiva-M. Phytochem Anal 1999; 10: $22-5$

57 Siebert DJ. Localization of salvinorin A and related compounds in glandular trichomes of the psychoactive sage, Salvia divinorum. Ann Bot (Lond) 2004; 93: $763-71$. 